\title{
Primary prevention of cardiovascular disease events with angiotensin-converting enzyme inhibitors and angiotensin receptor blockers in patients with autosomal dominant polycystic kidney disease on dialysis - a nationwide cohort study
}

Chien-Yu Lin

En Chu Kong Hospital

Chien-Lin Lu

Fu Jen Catholic University

Lian-Yu Lin

National Taiwan University

Pau-Chung Chen

national Taiwan university

Kuo-Cheng Lu

Fu Jen Catholic University

Dong-Feng Yeih ( $\square$ ocean0829@yahoo.com)

Catholic University of Korea School of Medicine Department of Internal Medicine https://orcid.org/0000-0003-4851-5663

\section{Research article}

Keywords: autosomal dominant polycystic kidney disease (ADPKD); angiotensin-converting enzymes inhibitor (ACEI); angiotensin II receptor blocker (ARB); renin-angiotensin-aldosterone system (RAAS) blockade; cardiovascular disease (CVD);

Posted Date: July 25th, 2019

DOI: https://doi.org/10.21203/rs.2.11873/v1

License: (c) (1) This work is licensed under a Creative Commons Attribution 4.0 International License. Read Full License 


\section{Abstract}

Background: Although renin-angiotensin-aldosterone system (RAAS) blockade has been shown to reduce cardiovascular disease (CVD) in the general population and high risk subjects, their protective effect in autosomal dominant polycystic kidney disease (ADPKD) patients under dialysis was still unknown. Methods: By using the database from 1995 to 2008 Taiwan National Health Insurance Research Database (Registry for Catastrophic Illnesses), we included 387 ADPKD patients who received dialysis therapy, aged $\geq 18$ year-old, and with no evidence of CVD events in 1997 and 1998 . We utilized Cox proportional hazards regression analysis and propensity score matching to evaluate adjusted hazard ratios for all-cause mortality and CVD events in users $(n=231)$ and nonusers $(n=156)$ of an angiotensinconverting enzymes inhibitor (ACEI) / angiotensin II receptor blocker (ARB) during the 12 years of followup. Results: All study subjects were followed up for more than 3 months. Compared with the control group, the ACEI/ARB treatment group did not have favorable outcome including acute coronary syndromes, receiving coronary intervention, cerebral vascular events, peripheral artery disease, heart failure and overall mortality. The results remain similar between groups before and after propensity score matching. Moreover, there was no significant difference in outcomes between ACEI/ARB treatment over $50 \%$ of follow-up period and without ACEI/ARB treatment after propensity score matching. Conclusions: We found ACEI or ARB usage is not associated with a reduction of cardiovascular events and survival benefit in our nationwide cohort study of ADPKD patient on dialysis from Taiwan. Further larger scale, multicenter and randomized control trials are warranted to show the causal association.

\section{Background}

Autosomal dominant polycystic kidney disease (ADPKD) is the most common hereditary cause of endstage renal disease (ESRD), accounting for 8-10\% of ESRD patients in western countries [1]. It occurs 1 of 400-1000 individuals $[2,3]$ and is characterized by development of cystic dilatation of renal tubules with progressive destruction of renal parenchyma $[4,5]$. As the cysts grow, $45 \%$ of patients will progress to end-stage renal disease (ESRD) by the age of 60 and up to $75 \%$ by the age of 70 [6]. Hypertension is very common in ADPKD, occurring in $50-70 \%$ of patients before renal function impairment $[7,8]$, and is associated with rapid progression to ESRD and adverse cardiovascular disease (CVD) outcomes [9]. Left ventricular diastolic dysfunction, endothelial dysfunction, and increased carotid artery intima-media thickness are found in young ADPKD patients who have normal blood pressure (BP) and renal function [10]. CVD complications have been a major cause of death in patients with ADPKD and the risk of CVD death is estimated to be 1.6-3.2-fold higher in these patients $[9,11]$.

Several mechanisms of cardiovascular dysfunction have been proposed in ADPKD patients, including activation of renin-angiotensin-aldosterone system (RAAS), impaired nitric oxide dependent vasodilatation, increased sympathetic nerve activity and plasma endothelin-1 concentration and insulin resistance $[12,13]$. Among them, RAAS activation plays an essential role. When compared with calcium channel blocker, RAAS blockade may decrease urinary albumin excretion in subjects with ADPKD [14]. 
However, uncertainty remains concerning the optimal choice of antihypertensive therapy in subjects with ADPKD in terms of in slowing progression to renal failure and CVD outcomes in ADPKD [14-17].

CVD is the leading cause of mortality in patients receiving dialysis $[18,19]$. In addition to fluid and sodium overload, dialysis patients are found to have increased sympathetic activity and abnormal response to RAAS, both of them result in higher incidence of hypertension and LVH. Although angiotensin-converting enzymes inhibitor (ACEI) and angiotensin II receptor blocker (ARB) have been shown to reduce CVD morbidity and mortality in the general population and high risk subjects [20-22], their protective effect in ESRD patients under dialysis remained uncertain [23]. Our previous study revealed that the overall mortality was significantly greater in patients who did not use an ACEI/ARB in ESRD patients on dialysis in a nationwide cohort [18]. In addition, subjects who used an ACEI/ARB for longer durations were significantly less likely to experience CVD events [18]. However, it remained unclear about the role of ACEI/ARB in primary prevention of CVD events in ADPKD patients with ESRD. To fill this gap, we hypothesize that the use of ACEI or ARB is associated with the reduction of major cardiovascular events in ADPKD patients on dialysis in a nationwide cohort.

\section{Methods}

\section{Study population}

By using the Registry for Catastrophic Illness database from a national health insurance program in Taiwan, we obtained demographic data, medication history, and diagnostic codes based on the International Classification of Diseases, Ninth Revision, Clinical Modification (ICD-9-CM; www.icd9data.com/2007) for the analyses. In the current study, we included ESRD subjects undergoing hemodialysis or peritoneal dialysis, and $\geq 18$ years between 1995 and 2008. We excluded subjects with history of acute coronary syndrome (ACS) or previous stroke before dialysis. All enrolled subjects were followed from 1995 to 2009 and the medium follow-up time was 1428 days. By reviewing of the pharmacy prescription database, we also gathered information on prescribed drugs, dosage, and duration. A total of 607 dialysis patients with ADPKD were enrolled and, among them, 193 patients were excluded if ever experienced with CVD before starting dialysis while 27 subjects were excluded due to follow-up less than 3 months. Finally, there were 387 subjects included in the final analyses. The designed patient flow diagram is shown in Figure 1. The study was approved by the Research Ethics Committee of the National Taiwan University Hospital, Taipei, Taiwan. All methods were carried out in accordance with relevant guidelines and regulations.

\section{Comorbidities and outcomes:}

After the index use of an ACEI or an ARB, we defined the comorbidities by searching the database for the presence of hypertension (ICD-9-CM codes: 401.X-405.X), diabetes mellitus (250.X, 249.X), hyperlipidemia (272.X), CVD events including coronary artery disease (411.X-414.X, V17.3, V81.0), atrial 
fibrillation (427.31, 427.3), valvular heart disease (394.X-396.X,) and liver cirrhosis (571.X, 572.X). The endpoints of the present study were death, new onset ACS (410.X, A270, 411.1), coronary intervention (CI): percutaneous coronary intervention $(00.66,36.0 X)$, ischemic stroke: (434.X, A293, A292), hemorrhagic stroke: (430.X, 431.X, 432.X), peripheral arterial disease $(250.7,443 . X, 444.2)$, heart failure $(428.0-428.3,428.9)$.

\section{Propensity score-based matching}

Propensity score (PS) matching is a statistical technique used to control the covariates to make two groups more comparable in observation study. In the current study, the PS dependent variable was receiving ACEI/ARB treatment or not. Other covariates, such as age, gender, hypertension, DM, dyslipidemia, comorbidities, and medications (antiplatelet, warfarin, beta-blocker, Statin), were put into a non-parsimonious logistic regression model. Participants were excluded from further analysis if an appropriate PS match could not be found. In the final analysis, the remaining subjects composed a matched $1: 1$ or $1: 2$ according to the original case number in each group.

\section{Statistical analysis}

All analyses were performed with SPSS 15.0 for WINDOWS 7 (SPSS Inc., Chicago, IL, USA). Student's t test was performed to compare continuous variables while chi-squared test was used to test categorical covariates. Fisher's exact test was used instead for categorical variable if any expected value within a $2 \times 2$ table was below 5. Cox's proportional hazard models to adjust covariates including age, gender, risk profile, and medications were used to estimate the risk of outcome associated with and without taking ACEI/ARB. The event-free survival time was defined as the time from the day of dialysis therapy to an endpoint. If an event did not occur, the case was regarded as censorship at the end of the study, withdraws from the insurance, loss contact, and receiving kidney transplantation. Moreover, we performed a subgroup analysis by including patients with more aggressive ACEI/ARB treatment (defined as ACEI/ARB treatment over $50 \%$ of the follow-up period) to test the consistency. Kaplan-Meier curves were performed to show the event-free survival trend between subjects with and without taking ACEI/ARB and tested by log-rank test. A $P$ value $<0.05$ was considered statistically significant in all analyses.

\section{Results}

\section{Demographic and clinical characteristic of enrolled patients}

The demographic and characteristic of the patients was shown in Table 1. Subjects treated with ACEI/ARB for more than 30 days served as the study group, and those treated with less than 30 days or no medication served as the control group. An ACEI or ARB was prescribed to 231 subjects $(59.7 \%)$. Subjects taking an ACEI or ARB were at higher prevalence of hypertension $(94.8 \%$ vs $73.7 \%, p<0.001)$ and 
more likely to receive a concomitant medication, including beta-blocker $(72.7 \%$ vs $37.8 \%, p<0.01)$ and statin $(28.1 \%$ vs $13.5 \%, p<0.001)$. Otherwise, there was significant difference in age, gender, risk profile of atherosclerosis and cardiovascular comorbidities, including coronary artery disease, atrial fibrillation and valvular heart disease between groups. During the study period, there were less ischemic stroke/TIA (1.3\% vs $5.8 \%, p=0.017)$ and acute limb ischemia events $(3.0 \%$ vs $8.3 \%, p=0.021)$ in the ACEI/ARB treatment group. In addition, the overall mortality was lower in the treatment group $(13.0 \%$ vs $21.8 \%, p=0.022)$.

The PS matching enrolled 80 patients in the ACEI/ARB treatment group and 160 patients without taking ACEl/ARB (Table 1). After PS matching, there were smaller differences in age, sex, comorbidities, use of other medication, and other clinical variables between two groups. After PS mating matching, the incidence of ischemic stroke/TIA was significantly lower in ACEI/ARB treatment group than control group ( $0.6 \%$ vs $5.0 \%, p=0.044)$. By contrast, there was no significant difference between groups in incidence of ACS or $\mathrm{Cl}(5.6 \%$ vs $5.0 \%, P=1.0)$, hemorrhagic stroke ( $5.0 \%$ vs $3.8 \%, P=0.756)$ and acute limb ischemia ( $3.8 \%$ vs. $5.0 \%, P=0.735$ ) and hospitalization due to heart failure (5.6\% vs $5.0 \%, P=1.0$ ), and acute limb ischemia ( $5.6 \%$ vs $6.3 \%, p=1.0)$. In addition, the overall mortality was similar in both group $(14.4 \%$ vs $18.8 \%, p=0.381$ ). No patients were lost to follow-up in these two groups.

\section{Effect of Duration of ACEI/ARB Use}

The hazard ratios for different clinical outcomes in study subjects were shown in Table 2. Compared with the control group, the ACEI/ARB treatment group did not have favorable outcome including ACS, receiving $\mathrm{Cl}$, cerebral vascular events, peripheral artery disease, heart failure and overall mortality. The results remain similar between groups before and after PS matching.

In Figure 2, we demonstrated the probability of survival in subjects receiving ACEI/ARB before and after PS-matching by the Kaplan-Meier curve. The results showed that before and after PS matching, patients received ACEI/ARB had similar survival rate (Figure 2A and 2C). In Fig. 2B and 2D, we showed the Kaplan-Meier curve for the cardiovascular event-free rate (ACS, Cl, ischemic stroke, transient ischemic accident, hemorrhagic stroke, peripheral artery disease, heart failure) before/after PS matching. The results showed there was no difference in composite endpoint between groups before and after PS matching.

\section{Characteristics of patients with ACEI/ARB treatment over $50 \%$ of follow-up period}

Table 3 analyses the demographic and clinical characteristic in ADPKD subjects on dialysis treated with ACEI/ARB for more than $50 \%$ of follow-up period. The results showed the patients who used ACEI/ARB had higher prevalent of hypertension $(95.5 \%$ vs $73.7 \%, p<0.001)$ and more likely to receive a concomitant medication, including beta-blocker $(74.5 \%$ vs $37.8 \%, p<0.01)$ and statin $(27.3 \%$ vs $13.5 \%$, $p<0.001)$. During the study period, there were less event of heart failure $(1.83 \%$ vs $8.3 \%, p=0.023)$ in the 
ACEI/ARB treatment group. There was no significant difference in overall mortality between ACEI/ARB treatment group and control group (12.7\% vs $21.8 \%, p=0.058)$. After PS matching, the ACEI/ARB group had similar heart failure event $(0.0 \%$ vs $6.5 \%, p=0.058)$, and the overall mortality was similar in both group $(13.0 \%$ vs $19.5 \%, p=0.275)$.

\section{Discussion}

To the best of our knowledge, this is the first study to investigate the impact of RAAS blockade on CVD events and all-cause mortality in ADPKD subjects on dialysis. Our study demonstrated that RAAS blockade treatment in ADPKD subjects on dialysis cannot significantly reduce the total mortality and CVD composite endpoint including acute coronary syndrome, ischemic stroke/TIA or hemorrhagic stroke and peripheral artery disease and hospitalization due to acute heart failure. To test the effect of treatment duration on CVD outcome, we compared subjects with ACEI/ARB treatment for more than $50 \%$ of follow up period with control subjects. Our data also showed that treatment duration exerted insignificant effects on total mortality and CVD outcomes.

In subjects with chronic kidney disease, RAAS blockade had been shown to slow renal function deterioration and reduce proteinuria $[15,17]$. In hypertensive ADPKD patients under similar BP control, subjects treated with diuretics had a faster loss of renal function than treated with ACEI, [24]. However, the benefit of ACEl in slowing renal function progression has not yet shown when compared to amlodipine and atenolol in ADPKD patients $[14,16,17]$. These studies failure to show the benefit of RAAS blockade in rate of glomerular filtration rate decline or ESRD progression and were limited by older patients enrolled and relative small number of study population. The results of two randomized, doubleblind, placebo-controlled trials have shown that dual therapy with ACEI and ARB was not more effective than either ARB or ACEl alone in slowing annual change in total kidney volume and renal function deterioration in both early and late ADPKD patients $[25,26]$. When compared with the standard BP control group, the aggressive BP control group was associated with a modest reduction in total kidney volume and improvement in LV mass index and urinary albumin excretion. Although the studies did not enroll a control group not taking an ACEl, it will continue as first-line medications in hypertensive ADPKD patients because of its safety and good BP control [27]. The results recalls the failure of tight BP control to slow renal function progression in the African-American Study of Kidney Disease and Hypertension trial [28], suggesting that earlier approach to the biology of the disease should be considered [27].

Higher left ventricular mass index can be found since early stage of ADPKD and is significantly associated with ambulatory systolic BP in normotensive and hypertensive ADPKD patients [29]. LVH is at the prevalence of around $48 \%$ among hypertensive and $23 \%$ among normotensive ADPKD patients. Hypertension is known to occur frequently and early in ADPKD and considered as a contributing factor for LVH in part due to earlier onset and inadequate treatment [30]. The HALT PKD study using cardiac magnetic resonance to assess LVH and reported ADPKD patients with less than 50 years of age and prior use of RAAS blockade have lower prevalence of $\mathrm{LVH}$, and therefore early BP intervention can decrease LVH progression and might potentially decrease CVD mortality [31]. In UK General Practice Research 
Database provide further evidence that early and aggressive BP control in ADPKD through increasing coverage and intensity of antihypertension therapy can effectively lower all-cause mortality in ADPKD patients [32]. However, the above study results did not provide evidence to suggest preferential benefit from ACEI/ARB treatment. Currently, there was no prospective randomized controlled trial investigating the effects of ACEI/ARB treatment on mortality and CVD events in ADPKD patients.

In general, ADPKD patients had better survival on dialysis than non-ADPKD patients [33]. There is a trend of the increase in the number of ADPKD patients being dependent on renal replacement therapy from 1990 s to 2000 s and is thought to be related to greatly improvement on dialysis survival rather than incidence of dialysis increase [34]. CVD, coronary artery disease and infection are the leading causes of death in ADPKD patient on dialysis [35,36]. Although several evidences have demonstrated that ACEI and ARB can effectively reduce the BP and offer cardioprotective effects on dialysis patients [18,37], there is no available literature discussing the effects of RAAS blockade on reducing the occurrence of CVD events and survival benefit on ADPKD patients on dialysis. In the current study, we found the ACEls and ARBs treatment was not associated with an overall reduction in CVD events and all-cause mortality in ADPKD patients under dialysis. However, a recent nationwide population-based cohort study showed that statin and RAAS blockade significantly reduced both hemorrhagic and ischemic strokes in ADPKD subjects without ESRD or cerebrovascular accident (CVA) [38]. It is possible that RAAS blockade should be given earlier during the course of progressive vascular damage. Further study is warranted to elucidate the effect of RAAS blockade in ADPKD patients under dialysis.

The main strengths of this present study are two-fold. First, our present study was a population-based, nationwide study that recruited all validated dialysis patients in Taiwan and followed them for a 12-year period. Second, all medications and comorbidities were recorded under the national health insurance policy. Our study also had several imitations. First, we exclusively relied on claim data, so there may be a bias in disease classification. Second, other possible confounding factors for example, blood test including electrolyte imbalance, lipid profiles, and inflammation parameters, and nutritional status and vascular calcification data were not available to adjust the risk of cardiovascular events and mortality. Third, a limitation of PS matching was that it dealt with known confounding factors. Some unknown confounding factors, that might be unequally distributed in both treatment and control groups, might have affected the observed difference in our study.

\section{Conclusions}

In summary, in our nationwide cohort study of ADPKD patient on dialysis from Taiwan, we found ACE inhibitor or ARB usage is not associated with a reduction of cardiovascular events and survival benefit. Further larger scale, multicenter and randomized control trials are warranted to show the causal association. 


\section{Declarations}

Ethics approval and consent to participate: The study was approved by the Research Ethics Committee of the National Taiwan University Hospital, Taipei, Taiwan.

Consent for publication: Not applicable

Availability of data and material: The datasets used and/or analysed during the current study are available from the corresponding author on reasonable request.

Competing interest: The authors have no conflicts of interest to disclose.

Funding: There was no funding in this study.

Author's contributions: Designed the study, drafted the article, and interpretated the data: Chien-Yu Lin, and Chien-Lin Lu; helped conduct the literature review, approved the analytic strategy: Lian-Yu Lin; designed and performed the study's analytic strategy: Pau-Chung Chen, and Kuo-Cheng Lu; contributed to the study ' s conception, reviewed the study, and final approval of the version to be published: Dong-Feng Yeih.

Acknowledgments: We thank the many people who have contributed to the data we examined, including all of the anonymous participants in the study.

\section{References}

1. Perrone RD, Ruthazer R, Terrin NC: Survival after end-stage renal disease in autosomal dominant polycystic kidney disease: contribution of extrarenal complications to mortality. American journal of kidney diseases : the official journal of the National Kidney Foundation 2001, 38(4):777-784.

2. Torres VE, Harris PC, Pirson Y: Autosomal dominant polycystic kidney disease. Lancet (London, England) 2007, 369(9569):1287-1301.

3. Johnson AM, Gabow PA: Identification of patients with autosomal dominant polycystic kidney disease at highest risk for end-stage renal disease. Journal of the American Society of Nephrology : JASN 1997, 8(10):1560-1567.

4. Watnick T, Germino GG: Molecular basis of autosomal dominant polycystic kidney disease. Seminars in nephrology 1999, 19(4):327-343.

5. Trujillano D, Bullich G, Ossowski S, Ballarin J, Torra R, Estivill X, Ars E: Diagnosis of autosomal dominant polycystic kidney disease using efficient PKD1 and PKD2 targeted next-generation sequencing. Molecular genetics \& genomic medicine 2014, 2(5):412-421.

6. Gabow PA, Johnson AM, Kaehny WD, Kimberling WJ, Lezotte DC, Duley IT, Jones RH: Factors affecting the progression of renal disease in autosomal-dominant polycystic kidney disease. Kidney international 1992, 41(5):1311-1319. 
7. Chapman AB, Schrier RW: Pathogenesis of hypertension in autosomal dominant polycystic kidney disease. Seminars in nephrology 1991, 11(6):653-660.

8. Ecder T, Schrier RW: Hypertension in autosomal-dominant polycystic kidney disease: early occurrence and unique aspects. Journal of the American Society of Nephrology : JASN 2001, 12(1):194-200.

9. Ecder T: Cardiovascular complications in autosomal dominant polycystic kidney disease. Current hypertension reviews 2013, 9(1):2-11.

10. Ecder T, Schrier RW: Cardiovascular abnormalities in autosomal-dominant polycystic kidney disease. Nature reviews Nephrology 2009, 5(4):221-228.

11. Luciano RL, Dahl NK: Extra-renal manifestations of autosomal dominant polycystic kidney disease (ADPKD): considerations for routine screening and management. Nephrology, dialysis, transplantation : official publication of the European Dialysis and Transplant Association - European Renal Association 2014, 29(2):247-254.

12. Loghman-Adham $M$, Soto $C E$, Inagami $T$, Cassis $L$ : The intrarenal renin-angiotensin system in autosomal dominant polycystic kidney disease. American journal of physiology Renal physiology 2004, 287(4):F775-788.

13. Lin CF, Chan HL, Hsieh YH, Liang HY, Chiu WC, Huang KY, Lee Y, Mclntyre RS, Chen VC: Endometrial cancer and antidepressants: A nationwide population-based study. Medicine 2016, 95(29):e4178.

14. Ecder T, Chapman AB, Brosnahan GM, Edelstein CL, Johnson AM, Schrier RW: Effect of antihypertensive therapy on renal function and urinary albumin excretion in hypertensive patients with autosomal dominant polycystic kidney disease. American journal of kidney diseases : the official journal of the National Kidney Foundation 2000, 35(3):427-432.

15. Bakris GL: Slowing nephropathy progression: focus on proteinuria reduction. Clinical journal of the American Society of Nephrology : CJASN 2008, 3 Suppl 1:S3-10.

16. Zeltner R, Poliak R, Stiasny B, Schmieder RE, Schulze BD: Renal and cardiac effects of antihypertensive treatment with ramipril vs metoprolol in autosomal dominant polycystic kidney disease. Nephrology, dialysis, transplantation : official publication of the European Dialysis and Transplant Association - European Renal Association 2008, 23(2):573-579.

17. Maschio G, Alberti D, Janin G, Locatelli F, Mann JF, Motolese M, Ponticelli C, Ritz E, Zucchelli P: Effect of the angiotensin-converting-enzyme inhibitor benazepril on the progression of chronic renal insufficiency. The Angiotensin-Converting-Enzyme Inhibition in Progressive Renal Insufficiency Study Group. The New England journal of medicine 1996, 334(15):939-945.

18. Wu CK, Yang YH, Juang JM, Wang YC, Tsai CT, Lai LP, Hwang JJ, Chiang FT, Chen PC, Lin JL et al: Effects of angiotensin converting enzyme inhibition or angiotensin receptor blockade in dialysis patients: a nationwide data survey and propensity analysis. Medicine 2015, 94(3):e424.

19. Sarnak MJ, Levey AS, Schoolwerth AC, Coresh J, Culleton B, Hamm LL, McCullough PA, Kasiske BL, Kelepouris E, Klag MJ et al: Kidney disease as a risk factor for development of cardiovascular disease: a statement from the American Heart Association Councils on Kidney in Cardiovascular 
Disease, High Blood Pressure Research, Clinical Cardiology, and Epidemiology and Prevention. Circulation 2003, 108(17):2154-2169.

20. Flather MD, Yusuf S, Kober L, Pfeffer M, Hall A, Murray G, Torp-Pedersen C, Ball S, Pogue J, Moye L et al: Long-term ACE-inhibitor therapy in patients with heart failure or left-ventricular dysfunction: a systematic overview of data from individual patients. ACE-Inhibitor Myocardial Infarction Collaborative Group. Lancet (London, England) 2000, 355(9215):1575-1581.

21. Mathew J, Sleight P, Lonn E, Johnstone D, Pogue J, Yi Q, Bosch J, Sussex B, Probstfield J, Yusuf S: Reduction of cardiovascular risk by regression of electrocardiographic markers of left ventricular hypertrophy by the angiotensin-converting enzyme inhibitor ramipril. Circulation 2001, 104(14):16151621.

22. Yusuf S, Sleight P, Pogue J, Bosch J, Davies R, Dagenais G: Effects of an angiotensin-convertingenzyme inhibitor, ramipril, on cardiovascular events in high-risk patients. The New England journal of medicine 2000, 342(3):145-153.

23. Akbari A, Knoll G, Ferguson D, McCormick B, Davis A, Biyani M: Angiotensin-converting enzyme inhibitors and angiotensin receptor blockers in peritoneal dialysis: systematic review and metaanalysis of randomized controlled trials. Peritoneal dialysis international : journal of the International Society for Peritoneal Dialysis 2009, 29(5):554-561.

24. Ecder T, Edelstein CL, Fick-Brosnahan GM, Johnson AM, Chapman AB, Gabow PA, Schrier RW: Diuretics versus angiotensin-converting enzyme inhibitors in autosomal dominant polycystic kidney disease. American journal of nephrology 2001, 21(2):98-103.

25. Schrier RW, Abebe KZ, Perrone RD, Torres VE, Braun WE, Steinman TI, Winklhofer FT, Brosnahan G, Czarnecki PG, Hogan MC et al: Blood pressure in early autosomal dominant polycystic kidney disease. The New England journal of medicine 2014, 371(24):2255-2266.

26. Torres VE, Abebe KZ, Chapman AB, Schrier RW, Braun WE, Steinman TI, Winklhofer FT, Brosnahan G, Czarnecki PG, Hogan MC et al: Angiotensin blockade in late autosomal dominant polycystic kidney disease. The New England journal of medicine 2014, 371(24):2267-2276.

27. Ellison $\mathrm{DH}$, Ingelfinger JR: A quest-halting the progression of autosomal dominant polycystic kidney disease. The New England journal of medicine 2014, 371(24):2329-2331.

28. Appel LJ, Wright JT, Jr., Greene T, Agodoa LY, Astor BC, Bakris GL, Cleveland WH, Charleston J, Contreras G, Faulkner ML et al: Intensive blood-pressure control in hypertensive chronic kidney disease. The New England journal of medicine 2010, 363(10):918-929.

29. Valero FA, Martinez-Vea A, Bardaji A, Gutierrez C, Garcia C, Richart C, Oliver JA: Ambulatory blood pressure and left ventricular mass in normotensive patients with autosomal dominant polycystic kidney disease. Journal of the American Society of Nephrology : JASN 1999, 10(5):1020-1026.

30. Chapman AB, Johnson AM, Rainguet S, Hossack K, Gabow P, Schrier RW: Left ventricular hypertrophy in autosomal dominant polycystic kidney disease. Journal of the American Society of Nephrology : JASN 1997, 8(8):1292-1297. 
31. Perrone RD, Abebe KZ, Schrier RW, Chapman AB, Torres VE, Bost J, Kaya D, Miskulin DC, Steinman TI, Braun $W$ et al: Cardiac magnetic resonance assessment of left ventricular mass in autosomal dominant polycystic kidney disease. Clinical journal of the American Society of Nephrology : CJASN 2011, 6(10):2508-2515.

32. Patch C, Charlton J, Roderick PJ, Gulliford MC: Use of antihypertensive medications and mortality of patients with autosomal dominant polycystic kidney disease: a population-based study. American journal of kidney diseases : the official journal of the National Kidney Foundation 2011, 57(6):856862.

33. Shaw C, Simms RJ, Pitcher D, Sandford R: Epidemiology of patients in England and Wales with autosomal dominant polycystic kidney disease and end-stage renal failure. Nephrology, dialysis, transplantation : official publication of the European Dialysis and Transplant Association - European Renal Association 2014, 29(10):1910-1918.

34. Spithoven EM, Kramer A, Meijer E, Orskov B, Wanner C, Caskey F, Collart F, Finne P, Fogarty DG, Groothoff JW et al: Analysis of data from the ERA-EDTA Registry indicates that conventional treatments for chronic kidney disease do not reduce the need for renal replacement therapy in autosomal dominant polycystic kidney disease. Kidney international 2014, 86(6):1244-1252.

35. Torra R, Darnell A, Cleries M, Botey A, Revert L, Vela E: Polycystic kidney disease patients on renal replacement therapy: data from the Catalan Renal Registry. Contributions to nephrology 1995 , 115:177-181.

36. Martinez V, Comas J, Arcos E, Diaz JM, Muray S, Cabezuelo J, Ballarin J, Ars E, Torra R: Renal replacement therapy in ADPKD patients: a 25-year survey based on the Catalan registry. $B M C$ nephrology 2013, 14:186.

37. Suzuki H, Kanno Y, Sugahara S, Ikeda N, Shoda J, Takenaka T, Inoue T, Araki R: Effect of angiotensin receptor blockers on cardiovascular events in patients undergoing hemodialysis: an open-label randomized controlled trial. American journal of kidney diseases : the official journal of the National Kidney Foundation 2008, 52(3):501-506.

38. Sung PH, Chiang HJ, Lee MS, Chiang JY, Yip HK, Yang YH: Combined renin-angiotensin-aldosterone system blockade and statin therapy effectively reduces the risk of cerebrovascular accident in autosomal dominant polycystic kidney disease: a nationwide population-based cohort study. Oncotarget 2017, 8(37):61570-61582.

\section{Tables}

Due to technical limitations, tables are only available as a download in the supplemental files section.

\section{Figures}


Figure 1

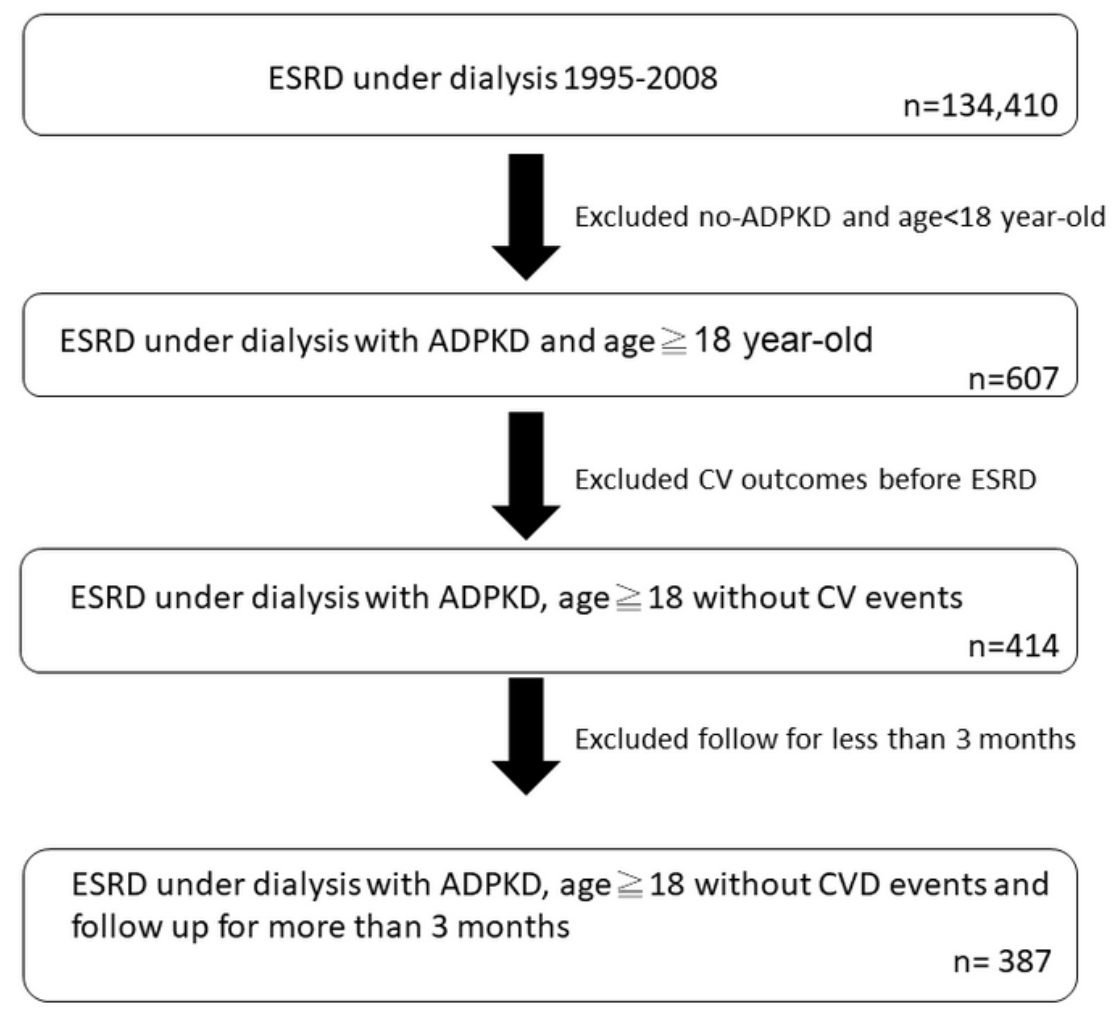

\section{Figure 1}

Designed patient flow diagram. Abbreviations: ADPKD, autosomal dominant polycystic kidney disease; CVD events, Cardiovascular disease events; ESRD, End-stage renal disease.

Figure 2

A

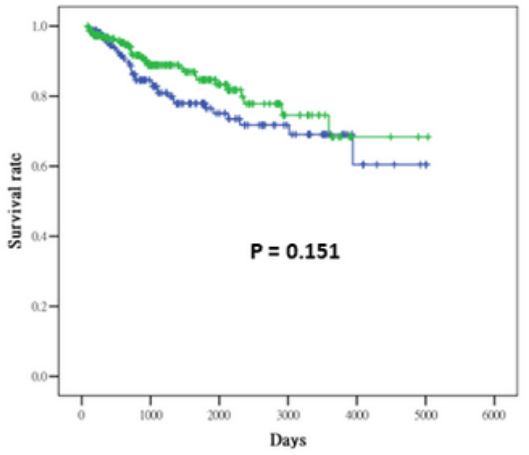

C

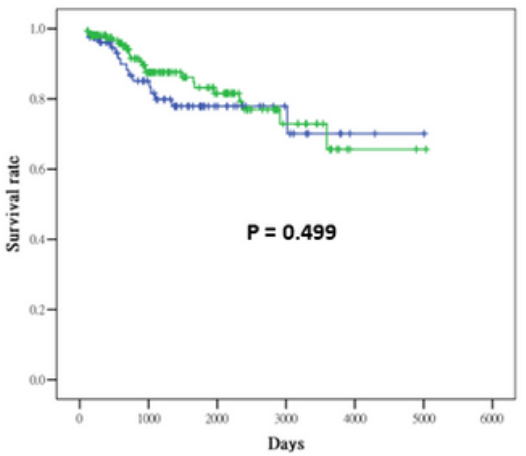

B

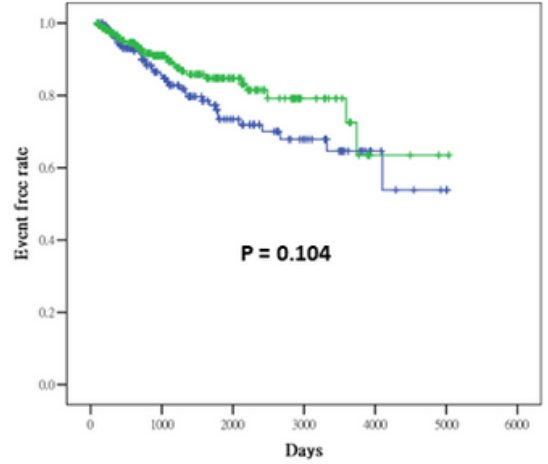

D

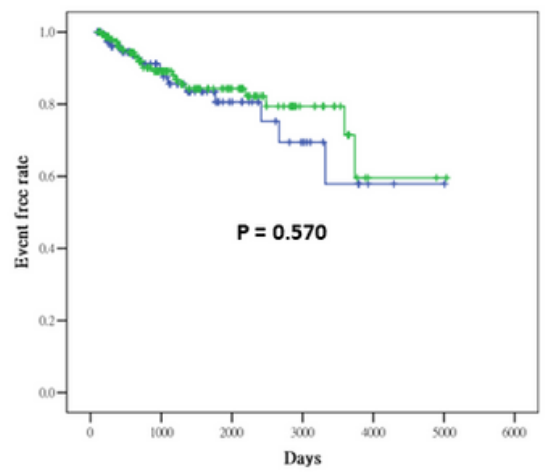




\section{Figure 2}

The Kaplan-Meier curve of survival (A) and cardiovascular events-free rate (B) between patients treated with (green) and without (blue) ACEI/ARB. The Kaplan-Meier curves of survival (C) and cardiovascular event-free rate (D) after PS matching were also illustrated. The cardiovascular events were defined as composite outcome of acute coronary syndrome, coronary intervention, ischemic stroke, transient ischemic accident, hemorrhagic stroke, peripheral artery disease.

\section{Supplementary Files}

This is a list of supplementary files associated with this preprint. Click to download.

- Tables.pdf 